\title{
A PROBLEM OF THERMAL SHOCK WITH THERMAL RELAXATION
}

\section{RAMAMURTHY and A. V. MANOHARA SHARMA}

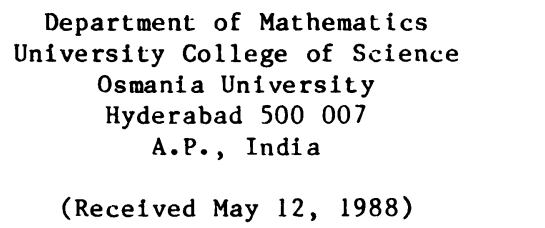

ABSTRACT. The problem of a semi-infinite medium subjected to thermal shock on its plane boundary is solved using the generalized theory of thermoelasticity. The expressions for temperature, strain and stress are presented. The results are exhibited graphically and compared with previous results.

KEY WORDS AND PHRASES. Generalized theory, relaxation time, Lame's elastic constants, diffusion equation, relaxation constant. 1980 AMS SUBJECT CLASSIFICATION CODE. 73U.

\section{INTRODUCTION.}

Thermoelastic problems are solved using the governing dynamical equations for the displacement and temperature. These equations are two partial differential equations.

Equation of motion:

$$
\ddot{u}_{i}=(\lambda+\mu) u_{j, i j}+\mu u_{i, j j}-(3 \lambda+2 \mu) \alpha T,{ }_{1} \cdot
$$

Equation of energy:

$$
k T_{11}=\rho C_{e} \dot{T}+(3 \lambda+2 \mu) \alpha T_{0} \dot{\varepsilon}_{k k} \text {. }
$$

The first equation is of wave type and the other is of diffusion type. For an isotropic, homogeneous elastic body subjected to a shock, the latter equation shows that the disturbance will be felt instantaneously at distances far from its source. As the equations are coupled this effect will be felt in both temperature and displacement. Such a behavior is physically inadmissible and contradicts the existing theory of heat conduction.

Many researchers, for example Morse and Feshbach [1], Boley [2], Baumister and Hamill [3] have discussed this paradox and suggested some modifications in the governing equations. Lord and Shulman [4] proposed the Generalized theory of thermoelasticity, where the time lag needed for the onset of thermal wave-relaxation 
time is considered and is well-known as the modified coupled heal conduction equation. Based on this modified theury, considerable work is being done by many authors (Fox [5], Ignaczak [6], Sherief and Anwar [7], and Choudhuri and Sain [8]).

Here the problem of an isolropic, hornogeneous half-space subjected to thermal shock on its plane boundary is solved using the Laplace transform Lechnique. The equations concerning the generalized theory of thermoelasticily are used to solve the said problem. The boundary condition for lemperature is in the form of exponential heating, a more realistic situation. After effecting the laplace inversion, the expressions for temperature, strain and stress are obtained. As a special case, the results due to Danilovskaya [9] for step-type boundary conditions and that of Sternburg et al [10] for ramp type boundary condition can be oblained. Further by setting relaxation constant to zero, the resulls due to Daimaruya et al [11] are oblained.

\section{FORMULATION OF THE PROBLEM.}

Consider an isotropic homogeneous half space, subjected to a thermal disturbance on its boundary. The governing equations of the generalized theory of thermoelasticily for the one dimensional case, are

$$
\begin{aligned}
& \mathrm{k} \frac{\partial^{2} \mathrm{~T}}{\partial \mathrm{x}^{2}}=\rho \mathrm{C}_{\mathrm{e}}\left(\frac{\partial \mathrm{T}}{\partial t}+\tau_{0} \frac{\partial^{2} \mathrm{~T}}{\partial \mathrm{t}^{2}}\right)+(3 \lambda+2 \mu) \alpha \mathrm{T}_{0}\left(\frac{\partial^{2} \mathrm{u}}{\partial \mathrm{x} \partial \mathrm{t}}+\tau_{0} \frac{\partial^{3} \mathrm{u}}{\partial \mathrm{x} \partial \mathrm{t}^{2}}\right) \\
& \sigma_{\mathrm{xx}}=(\lambda+2 \mu) \frac{\partial \mathrm{u}}{\partial \mathrm{x}}-(3 \lambda+2 \mu) \alpha\left(\mathrm{T}-\mathrm{T}_{\mathrm{o}}\right) \\
& (\lambda+2 \mu) \frac{\partial^{2} \mathrm{u}}{\partial \mathrm{x}^{2}}-(3 \lambda+2 \mu) \alpha \frac{\partial T}{\partial \mathrm{x}}=\rho \frac{\partial^{2} \mathrm{u}}{\partial \mathrm{t}^{2}}
\end{aligned}
$$

where $k, \rho, \mathrm{C}_{e}, \alpha, \tau_{0}$ are thermal conductivity, density, specific heat, coefficient of linear thermal expansion and the relaxation time, respectively. $\lambda$ and $\mu$ are the well known Lame's elastic constants. Here $T, \sigma_{x x}$, and $u$ are temperature, stress and displacement, respectively.

The inftal and boundary conditions are

$$
\begin{aligned}
& T(x, 0)=T_{0}, x>0 \\
& u(x, 0)=0=\left(\frac{\partial u}{\partial t}\right)_{t=0},\left(\frac{\partial T}{\partial t}\right)_{t=0}=0 \\
& T(0, t)=T_{0}\left[1-\exp \left(-t / t_{0}\right)\right] \\
& u^{\prime}(0, t)=\frac{(3 \lambda+2 \mu) \alpha T_{0}}{(\lambda+2 \mu)}\left[1-\exp \left(-t / t_{0}\right)\right] .
\end{aligned}
$$

In the above, $T_{0}$, and $t_{0}$ are constants. The step type boundary condition is obtained when $t_{0}=0$, i.e. $T(0, t)=H(t)$, and the ramp type boundary condition is obtained by expanding the exponential type and neglecting the higher order terms. Here $H(t)$ is Heaviside unit step function.

The regularity boundary conditions are

$$
T(x, t), u(x, t), \sigma_{x x}(x, t) \rightarrow 0 \text { as } x \rightarrow \infty \text {. }
$$


Introducing the following non-dimensional variables

$$
\begin{aligned}
& z=\left(\frac{\lambda+2 \mu}{\rho}\right)^{1 / 2}-\frac{\rho C_{e}}{k} x, y=\frac{\lambda+2 \mu}{\rho} \frac{\rho C_{e}}{k}-t \text {, } \\
& \theta=\frac{\mathrm{T}-\mathrm{T}_{0}}{\mathrm{~T}_{0}}, \quad \sum=\sigma_{\mathrm{xx}}(3 \lambda+2 \mu) \alpha \mathrm{T}_{0} \\
& U=\left[\rho\left(\frac{\lambda+2 \mu}{\rho}\right)^{3 / 2} \frac{1}{(3 \lambda+2 \mu) \alpha T_{0}} \stackrel{\rho C}{k}-e^{e}\right] u
\end{aligned}
$$

for distance, time, temperature, stress, and displacement respectively in equations $(2.1)-(2.3)$, we get

$$
\begin{aligned}
& \Theta^{\prime \prime}-\theta^{\prime}-\ddot{B}=\bar{e}\left(\dot{U^{\prime}}+\ddot{B} \ddot{U}^{\prime}\right), \\
& U^{\prime \prime}-\Theta^{\prime}=\ddot{U} \text {, } \\
& \Sigma=U^{\prime}-\Theta
\end{aligned}
$$

where 'dot' and 'dash' denote differentiation with respect to $y$ and $z$ respectively and

$$
\beta=\left(\frac{\lambda+2 \mu}{\rho}\right) \frac{\rho C}{k} \cdot \tau_{o}, \bar{e}=\frac{(3 \lambda+2 \mu)^{2} \alpha^{2} T_{o}}{(\lambda+2 \mu)} \frac{\rho C_{e}}{(\lambda+2}
$$

Here $\beta$ and $\bar{e}$ are the relaxation constant and thermoelastic coupling constant, respectively.

In (2.8a), the strain acceleration term $\left(\ddot{U}^{\prime}\right)$ can be ignored as the product $\bar{e} \cdot \beta$ is much less than $\overline{\mathrm{e}}$ or $\beta$ in the intermediate and room temperature.

Now our initial and boundary conditions $(2.4)-(2.6)$ reduce to

$$
\begin{aligned}
& \Theta(z, 0)=U(z, 0)=\left(\frac{\partial U}{\partial y}\right)_{y=0}=0=\left(\frac{\partial \Theta}{\partial y}\right)_{y=0}, \\
& \Theta(0, y)=-\exp \left(-y / \tau_{0}^{\prime}\right) \\
& U^{\prime}(0, y)=1-\exp \left(-y / \tau^{\prime}{ }_{0}\right) \\
& \Theta(z, y), U(z, y), \Sigma(z, y)+0 \text { as } z \rightarrow \infty
\end{aligned}
$$

where

$$
\tau^{\prime}{ }_{0}=\left(\frac{\lambda+2 \mu}{\rho}\right) \frac{\rho C_{e}}{k} t_{0} \text {. }
$$

3. SOLUTION OF THE PROBLEM.

In order to obtain the solutions for the temperature ( $\theta$ ) and strains (U'), we first eliminate $U, \theta$ from the first two equations of (2.8), namely

$$
\begin{gathered}
\theta^{\prime \prime \prime}-(1+\beta) \ddot{\theta^{\prime \prime}}-(1+\overline{\mathrm{e}}) \dot{\theta^{\prime \prime}}+\ddot{\theta}+\beta \ddot{\theta}=0 \\
\mathbf{U}^{\prime \prime \prime}-(1+\beta) \mathrm{U}^{\prime \prime}-(1+\overline{\mathrm{e}}) \dot{U^{\prime \prime}}+\ddot{U}+\beta \ddot{U} \dot{U}=0 .
\end{gathered}
$$




$$
\begin{aligned}
& \bar{\theta}^{\prime \prime \prime}-p[p(1+\beta)+(1+\bar{e})] \bar{\theta}^{\prime \prime}+p^{2}\left(p+\beta p^{2}\right) \bar{\theta}=0 \\
& \bar{U}^{\prime \prime \prime}-p[p(1+\beta)+(1+\bar{e})] \bar{U}^{\prime \prime}+p^{2}\left(p+\beta p^{2}\right) \bar{U}=0 .
\end{aligned}
$$

Here $\bar{\theta}, \bar{U}$ are Laplace Lransforms of $O$ and $U$ respectively and $p$ is the Lransform parameter, and

$$
\begin{aligned}
& \bar{\theta}(0, p)=-\tau_{0}^{\prime} / p_{0} \\
& \bar{U} \cdot(0, p)=1 / p_{0} \\
& \bar{\theta}(z, 0)=\bar{U}(z, 0)=0 .
\end{aligned}
$$

In the above,

$$
p_{0}=p \tau_{0}^{\prime}+1
$$

From (3.3) and (3.4), we get

$$
\begin{aligned}
& \bar{\theta}=A_{1} \exp \left(-\alpha_{2} z\right)+A_{2} \exp \left(-\alpha_{2} z\right) \\
& \bar{U}=B_{1} \exp \left(-\alpha_{1} z\right)+B_{2} \exp \left(-\alpha_{2} z\right)
\end{aligned}
$$

where

$$
\begin{aligned}
& A_{1,2}= \pm D\left[\bar{e}+\tau_{0}^{\prime}\left(\alpha_{2,1}^{2}-p p_{0}^{\prime}\right)\right] \\
& \alpha_{1,2} B_{1,2}=\mp D\left[\bar{e}-\tau_{0}^{\prime} p p_{0}^{\prime}+p-\alpha_{2,1}^{2} / p\right] \\
& \frac{1}{D}=p_{0}\left(\alpha_{1}^{2}-\alpha_{2}^{2}\right) \\
& p_{0}^{\prime}=1+\beta p \\
& \alpha_{1,2}^{2}=\frac{p}{2}\left[(1+B) p+(1+\bar{e}) \pm\left[((1+\beta) p+(1+\bar{e}))^{2}-4 p p_{0}^{\prime}\right]^{1 / 2}\right] .
\end{aligned}
$$

We consider a special case in which the relaxation constant $(\beta)$ is expressed in terms of the coupling constant $\bar{e}$, i.e. $\beta=\frac{1}{1+\bar{e}}$. For this value of $\beta$, we get

$$
\alpha_{1}^{2}=p^{2}+p / \beta, \alpha_{2}^{2}=\beta p^{2}
$$

and then the transformed temperature and strain become

$$
\begin{aligned}
\bar{\theta}(z, p)= & N(p)\left[\left(\bar{e}-\tau_{0}^{\prime} p\right) \exp \left(-\alpha_{1} z\right)-\left(p^{2} \tau_{0}^{\prime}(1-\beta)+\overline{e p}_{0}\right) \exp \left(-\alpha_{2} z\right)\right] \\
\bar{U} \cdot(z, p)= & N(p)\left[\left[\bar{e}+p\left(1-\beta-\tau_{0}^{\prime}\right)-\beta_{0} \tau^{\prime} p^{2}\right] \exp \left(-\alpha_{1} z\right)\right. \\
& \left.+\left(1+\tau_{0}^{\prime} p_{0}^{\prime} p\right) \exp \left(-\alpha_{2} z\right)\right]
\end{aligned}
$$




$$
N(p)=\frac{1}{p_{0}\left(p+b^{2}\right)(1-\beta)}
$$

and

$$
b^{2}=\overline{B(1}-\frac{1}{\beta)} .
$$

Inverting (3.7) and (3.8), we get

$$
\begin{aligned}
\theta=C_{1} I(0) & +C_{2} \exp \left(-y / \tau_{0}^{\prime}\right) I\left(1 / \tau_{0}^{\prime}\right)+C_{3} \exp \left(-b^{2} y\right) I\left(b^{2}\right) \\
& +C_{4} \emptyset_{1}\left(1 / \tau_{0}^{\prime}\right)+C_{5} \emptyset_{1}\left(b^{2}\right)+C_{6} \emptyset_{2}\left(1 / \tau_{0}^{\prime}\right)+C_{7} \emptyset_{2}\left(b^{2}\right)
\end{aligned}
$$

and

$$
\begin{aligned}
U^{\prime}=K_{1} I(\emptyset) & +K_{2} I\left(1 / \tau_{0}^{\prime}\right)+K_{3} I\left(b^{2}\right)+K_{4} \emptyset_{1}\left(1 / \tau_{0}^{\prime}\right) \\
& +K_{5} \emptyset_{1}\left(b^{2}\right)+K_{6} \emptyset\left(1 / \tau_{0}^{\prime}\right)+K_{7} \emptyset_{2}\left(b^{2}\right)-(\beta /(1-\beta))\left(e^{-z / 2 \beta}-1\right)
\end{aligned}
$$

where

$$
\begin{aligned}
& I(w)=\int_{0}^{y} z \exp \left[\left(w-\frac{1}{2 \beta}\right) \ell\right] F(z, \ell) H(\ell-z) d \ell \\
& C_{1}=(1-\beta) D_{1}, \quad C_{2}=b^{2} \tau_{0}^{\prime} D_{1} D_{2} \\
& C_{3}=-\beta\left(b^{2} \tau_{0}^{\prime}+\bar{e}\right) D_{1} D_{2}, \quad C_{4}=-C_{2} / D_{1} \\
& C_{5}=-C_{3} / D_{1}, \quad C_{6}=D_{2}, \quad C_{7}=-D_{3} \cdot D_{2} /(1-B), \\
& k_{1}=C_{1}, \quad K_{2}=b^{2}\left(\tau_{0}^{\prime}-\beta\right) D_{1} D_{2} \exp \left(-y / \tau_{0}^{\prime}\right) \\
& K_{3}=D_{1} D_{2} D_{3} b^{2} \exp \left(-b^{2} y\right) /(1-\beta), \quad K_{4}=\frac{-b^{2}\left(\tau_{0}^{\prime}-\beta\right)}{\tau_{0}^{\prime}} D_{2} \exp (-z / 2 \beta) \\
& K_{5}=b^{2} \beta D_{2} D_{3} \exp (-z / 2 \beta), \quad K_{6}=-D_{2} /\left(b^{2} \tau_{0}^{\prime}(1-\beta)^{2}\right) \\
& K_{7}=b^{2} \beta D_{2} D_{3} /(1-\beta)^{2}, \quad D_{1}=1 /\left(144 \beta^{2}\right) \\
& D_{2}=1 /\left(1-b^{2} \tau_{0}^{\prime}\right), \quad D_{3}=(1-\beta)^{2}+\tau_{0}^{\prime} \\
& \emptyset_{1}(w)=[1-\exp [(z-y) w]] H(y-z) / w, \emptyset_{2}(w)=[1-\exp [(\sqrt{\beta z}-y) w]] H(y-\sqrt{B z}) / w \\
& F(z, y)=I_{1}\left(z^{\prime} / 2 \beta\right) / D_{1} Z^{\prime} \\
& H(y-z)=0, \quad 0<y<z \\
& =1, \quad y>2
\end{aligned}
$$

and

$$
z^{\prime}=\left(y^{2}-z^{2}\right)^{1 / 2}
$$

Here $I_{1}$ is the modified Bessel funclion of $t i r i b$ kind. 
The expression for stress can be obtained fom (2.8c), (3.9) and (3.10). Taking $\tau_{0}^{\prime}=0$, recovers the results due to Danilovskay.1 [9] and selting $B=0$, recovers the results of Datmaruya [11].

\section{RESULTS AND DISCUSSION.}

The results for temperature, strain and slress distribulions are evalualed numerically and exhibited graphically in figures 1 to 3 , for a particular value of the relaxation constant $(B)$ given by $B=1 /(1+\bar{e})$. The transporl of thermal energy in the medium, i.e. either a diffusion process or a wave Like process depends on the magnitude of the relaxation constant. It was observed that at low temperatures the magnitude of the relaxation constant becomes significant and the energy equalion predicts a wave-type phenomenon. The magnitudes of the coupling and relaxation constants were calculated over a range of intermediate and high temperatures by Lord [12]. The values of the coupling paramter $\bar{e}$ are smaller than unity for most of the materials.

Her $=$ for the computation, the relaxation constant $(B)$ was taken as 0.98 and $0.7 t$ (the corresponding values of $\bar{e}$ are 0.1 and 0.31 respectively) and the values 0.25 , $0.5, l^{\prime}$ and 2 for the $\tau_{0}^{\prime}$. The time-dependence of the non-dimenstonal temperature $(\theta)$, strain $\left(U^{\prime}\right)$, and stress $(\Sigma)$ are depicted as a function of non-dimensional time $y$ at the non-dimensional distance $z=2$ for $\tau_{0}^{\prime}=0.5$.

As the relaxation constant increases the corresponding components of temperature, strain and stress decrease. The gap between these corresponding parameters increases, due to the effect of relaxation time unlike that of coupled theory. It may be mentioned that a similar phenomena was observed by Daimaruya [11] in case of coupled theory. Moreover due to presence of Heaviside unit step function, the two discontinuities can occur in temperature and stress at the wavefronts $y=z$ and $y=\bar{V}_{B} z$ and the corresponding accoustic velocity $\left(v_{1}\right)$ and thermal velocity $\left(v_{2}\right)$ at the wavefronts are 1 and $1 / \sqrt{\beta}$ respectively. Last but not least, the results obtained here which including the effect of the relaxation time are more general.

\section{ACKNOWLEDGEMENT.}

The second author wishes to thank the Council of Scientific and Industrial Research, New Delhi, for the financial assistance given for his work. 


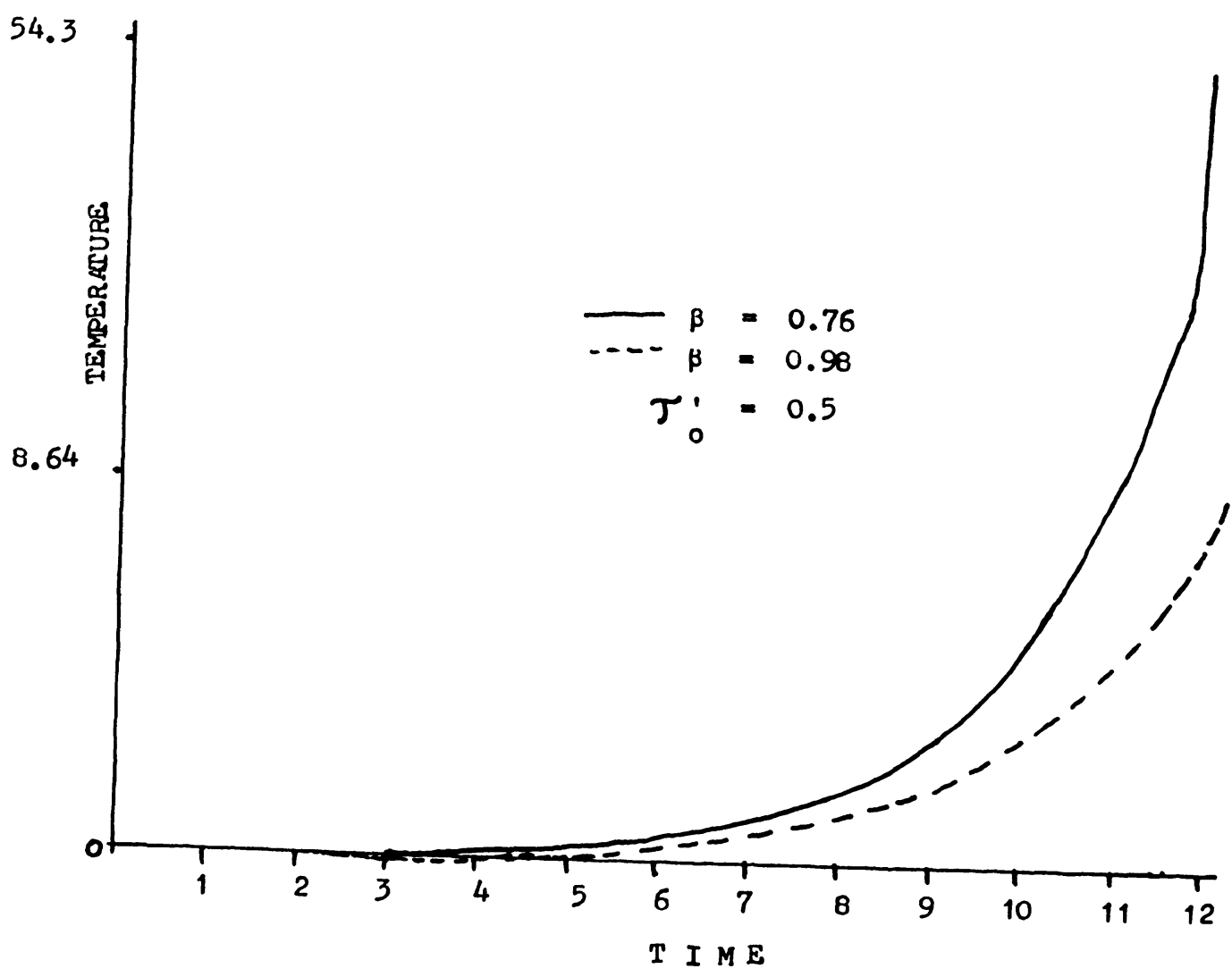

Fig. 1. Time dependence of temperature at $z=2$.

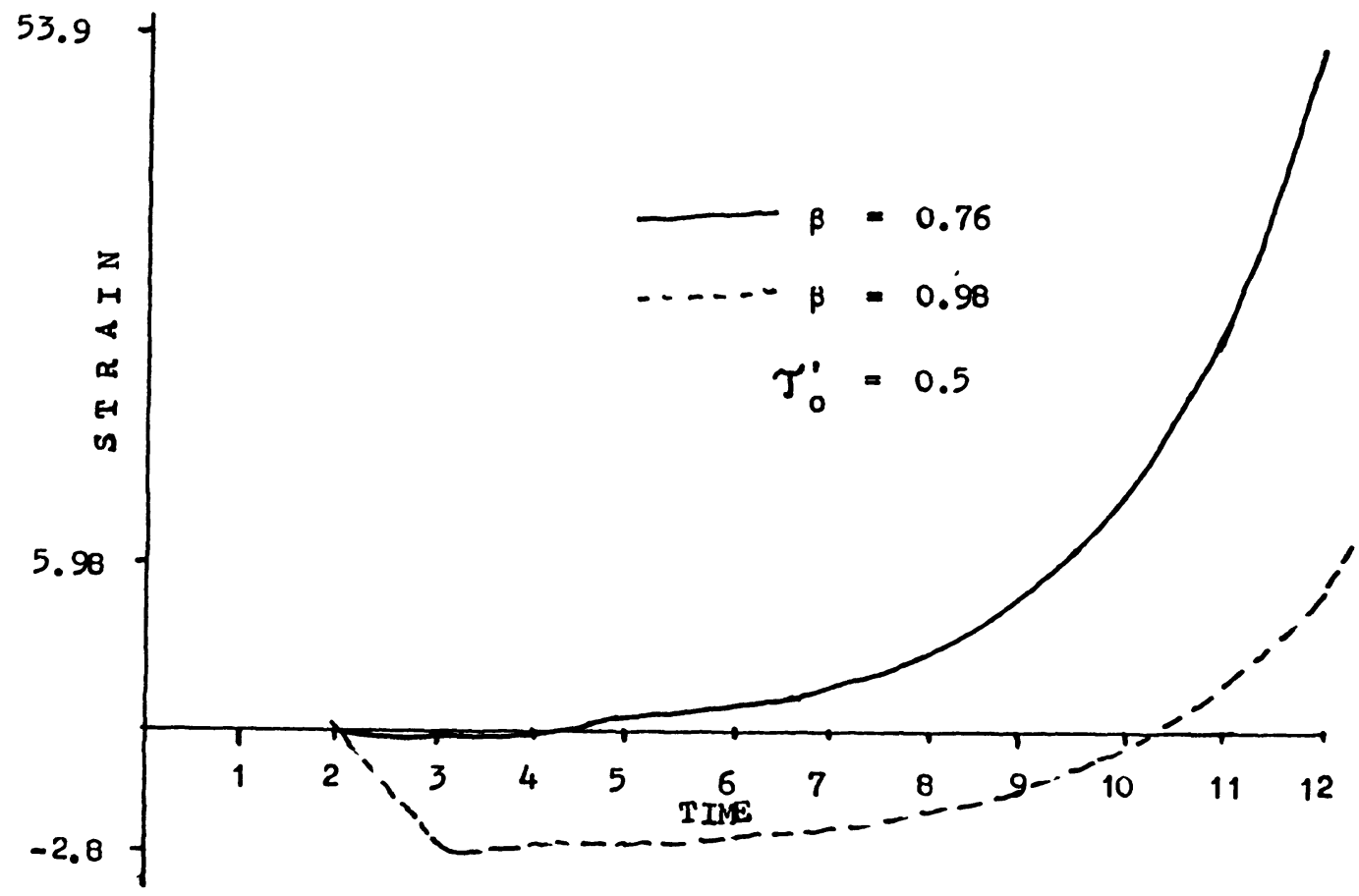

F1g. 2. Time dependence of strain at $z=2$. 


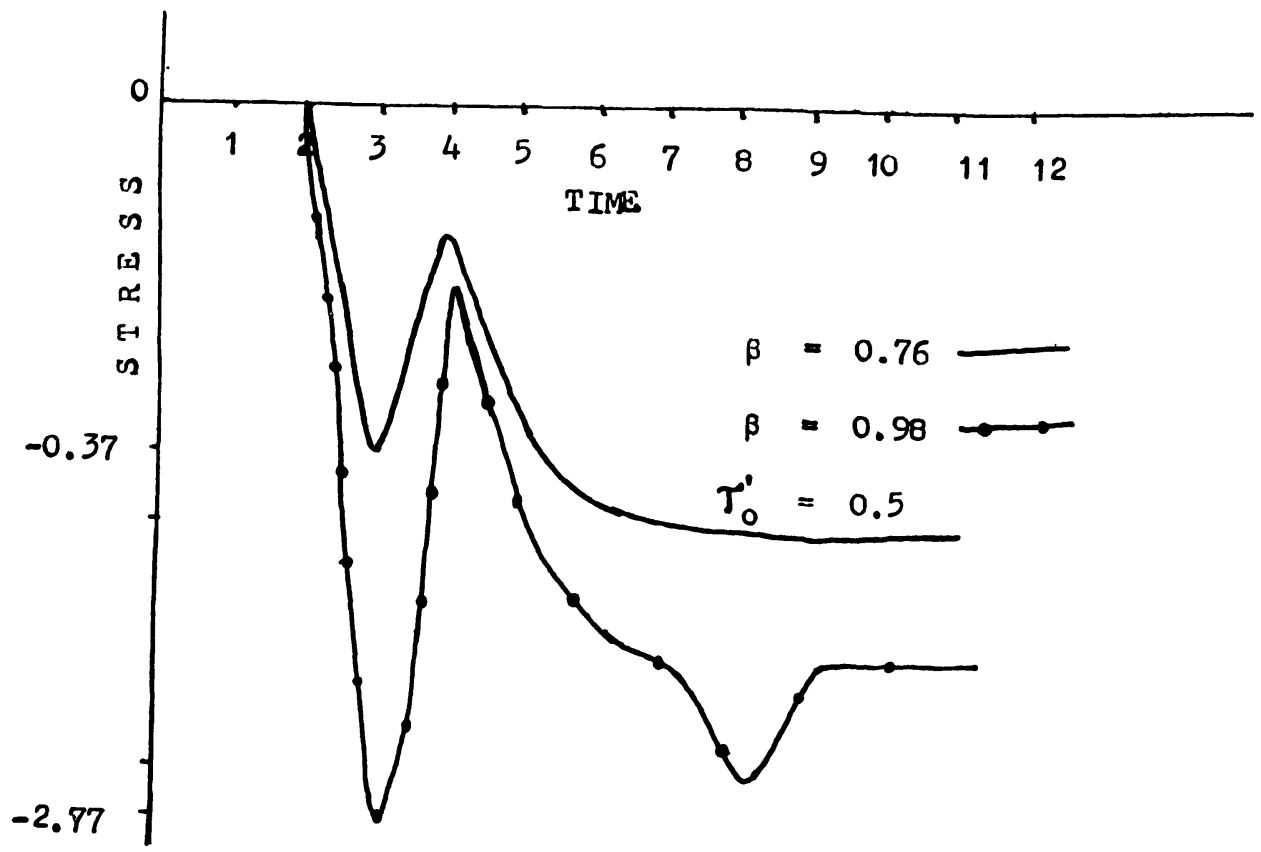

F1g. 3. Time dependence of stress at $z=2$.

REFERENCES

1. MORSE, P.M. and FESHBACH, H., Methods of Theoretical Physics, McGraw-Hill, New York, 1953.

2. BOLEY, B.A., The Analysis of Problems of Heat Conduction, Pergamon Press, New York, 1964.

3. BAUMISTER, K.J. and HAMILL, T.D., Hyperbolic Heat Conduction Equation, Jn1. of Heat Transfer, ASME Trans. 91 (1969), 543-548.

4. LORD, H.W. and SHULMAN, Y., A Generalized Dynamical Theory of Thermoelasticity, J. Mech. Phys. Solids 15 (1967), 299-309.

5. FOX, N., Generalized Thermoelasticity, Int. J. Engg. Sc1. 7 (1969), 437-445.

6. IGNACZAK, J., A strong discontinuity wave in Thermoelasticity with Relaxation Times, Jnl. of Thermal stresses 8 (1985), 25-40.

7. SHERIEF, H.H. and ANWAR, N., Problem in Generalized Thermoelasticity., Jnl. of Therma1 Stresses 9 (1986), 165-181.

8. ROY CHOUDHURI, S.K. and SAIN, G., Instantaneous Heat Sources...With Thermal relaxation., Ind. J. Pure. App1. Math. 13 (1982), 1340-1353.

9. DANILOVSKAYA, V.I., Thermal Stresses in an Elastic Half-space....Prikl. Mat. Mech. 14 (1950), 816-820.

10. STERNBERG, E. and CHAKRAVORTHY, J.G., On Inertia Effects in a Transient Thermoelastic Problem, Trans. ASME. Ser. E. 26 (1959), 503.

11. DAIMARUYA, M. and NATTO, N., A Transient Coupled Thermoelastic Problem in the Semi-infinite Medium. Bul1. JSME. 16 (1973), 1494-1505.

12. LORD, H.W., Ph.D. Dissertation, North Western University, Evanstan, 1966. 


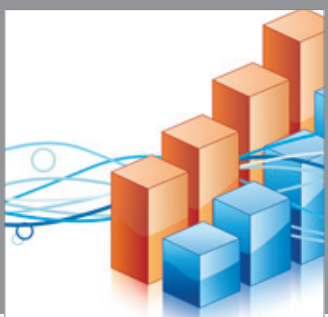

Advances in

Operations Research

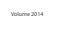

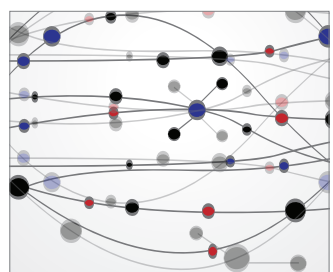

\section{The Scientific} World Journal
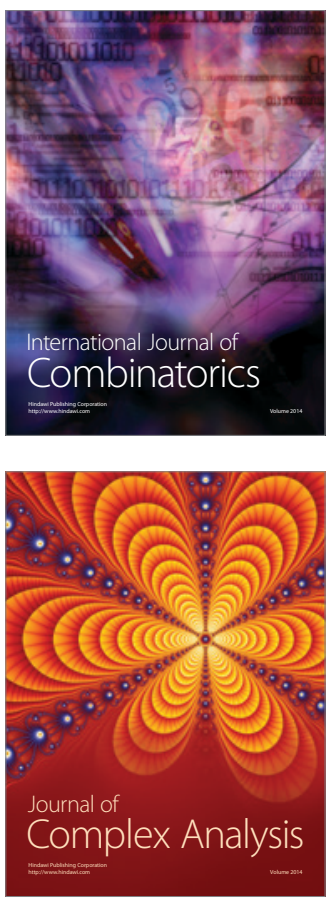

International Journal of

Mathematics and

Mathematical

Sciences
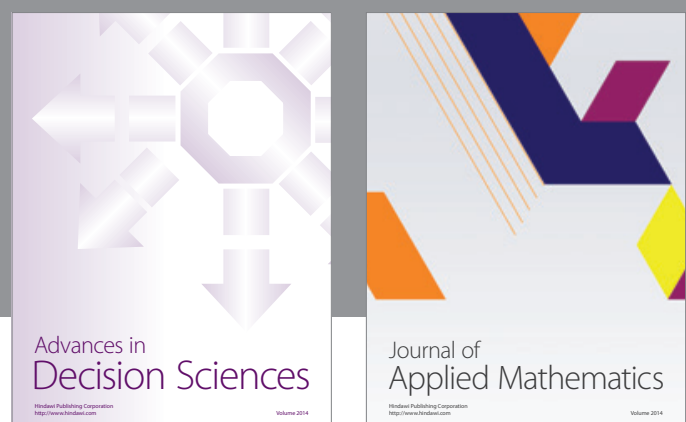

Journal of

Applied Mathematics
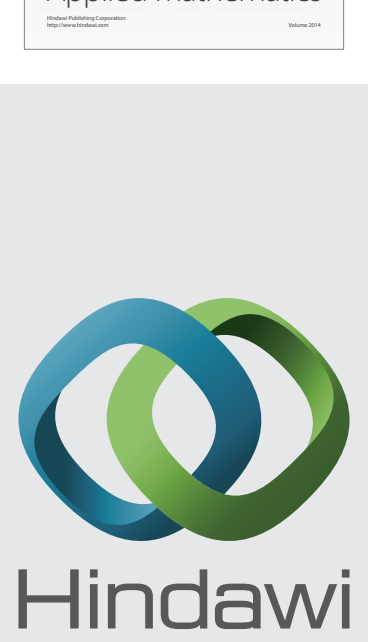

Submit your manuscripts at http://www.hindawi.com
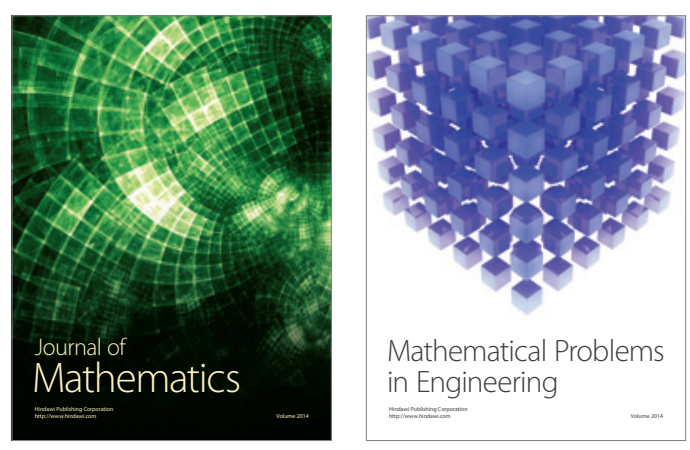

Mathematical Problems in Engineering
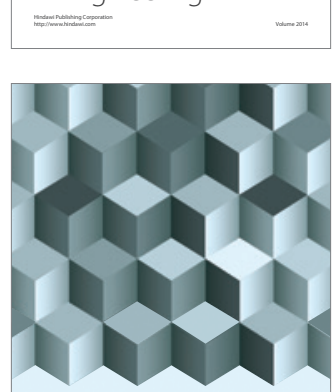

Journal of

Function Spaces
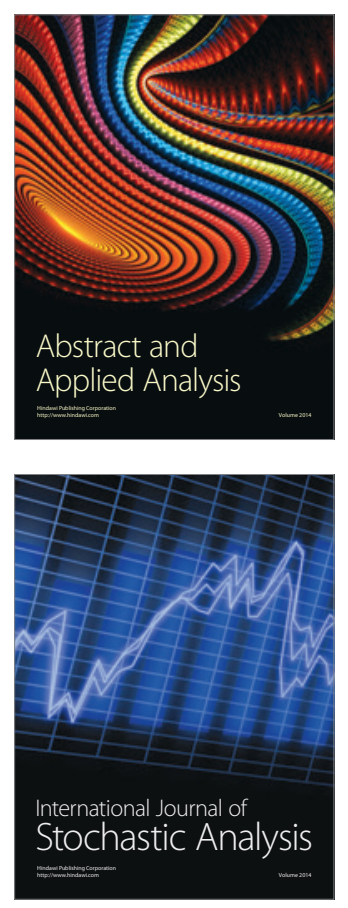

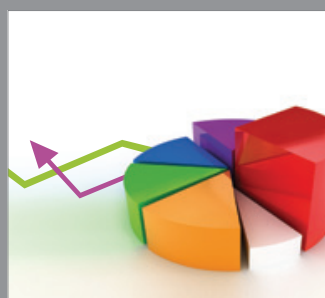

ournal of

Probability and Statistics

Promensencen
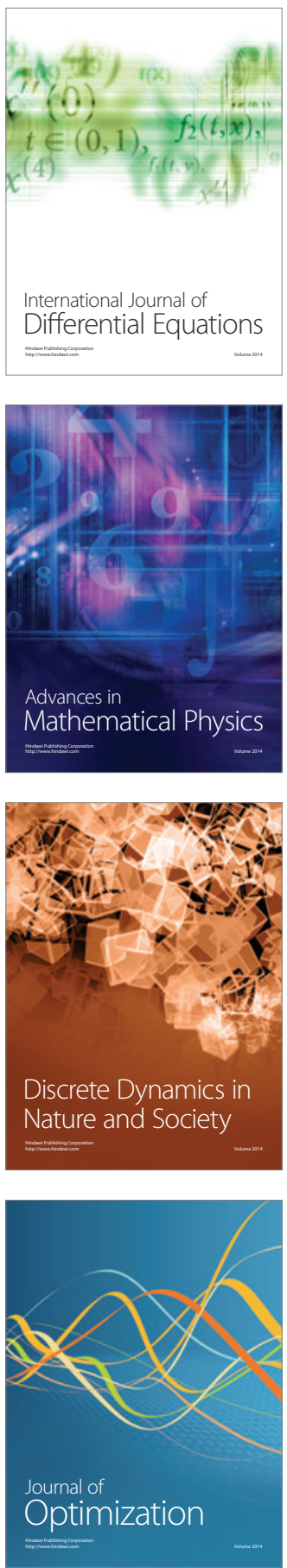